\title{
Charge correlations and strongly intensive fluctuations in ultrarelativistic nuclear collisions in the string model
}

\author{
Vladimir Kovalenko' \\ Saint Petersburg State University \\ Universitetskaya nab., 7-9, 199034, St. Petersburg, Russia \\ E-mail: v.kovalenko@spbu.ru
}

The fluctuations of the net electric charge of hadrons, produced in ultrarelativistic heavy ion collisions are considered in the Monte Carlo model with string fusion. We demonstrate that the observed experimentally centrality dependence of the dynamical net charge fluctuation variable can be explained by formation in central AA collisions of the strings of higher string tension. We provide the predictions for net charge correlations in $\mathrm{Pb}-\mathrm{Pb}$ collisions at $\mathrm{LHC}$ energy and discuss the applicability of the method at SPS and NICA energies.

XIII Quark Confinement and the Hadron Spectrum - Confinement 2018

31 July - 6 August 2018

Maynooth University, Ireland

${ }^{1}$ Speaker 


\section{Introduction}

The fluctuations of the net electric charge of hadrons, produced in ultrarelativistic heavy ion collisions, were proposed as one of the indicators of the formation of a quark-gluon plasma $[1,2]$. They also carry important information on the collective dynamical effects in AA collisions $[3,4]$.

Experimentally, they are studied in terms of dynamical fluctuation parameter $v_{\mathrm{dyn}}$ and the balance functions. These observables showed to be robust against volume fluctuations and centrality class width, being therefore strongly intensive variables [5].

The comparison of theoretical predictions of the net charge fluctuations, initially made in statistical models $[3,6,7]$, does not allow unambiguous conclusions about the formation of quark-gluon plasma in ultrarelativistic heavy ion collisions at RHIC and LHC. It was shown [8] that the experimental behavior of the net charge fluctuations, including the dependence on the width of the pseudorapidity window, is successfully described by the string model, and its dependence on centrality is related to the average string tension. For the more detailed study, the method of net charge long-range correlations in the windows separated by rapidity has been proposed for the better exclusion the short-range correlation effects [8].

In this paper we calculate the strongly intensive correlations and fluctuations of produced hadrons in a string-partonic Monte Carlo model [9, 10], taking into account fusion of quarkgluon strings [11,12], finite rapidity width of strings and explicit charge conservation. The model successfully describes the main features of forward-backward correlations between multiplicities and transverse momentum in $\mathrm{pp}$ and $\mathrm{Pb}-\mathrm{Pb}$ collisions at LHC energy $[13,14]$. We demonstrate that the centrality dependence of the width of the dynamical net charge fluctuation can be explained by formation in central AA collisions of the strings of higher string tension. We provide the predictions for net charge correlations in $\mathrm{Pb}-\mathrm{Pb}$ collisions at LHC energies and discuss the applicability of the method at SPS and NICA energies.

\section{Model description}

The present model describes nucleon-nucleon collisions on the partonic level. For the nuclear density of the $\mathrm{Pb}$ nuclei the Woods-Saxon distribution is taken. Each nucleon is considered as a set of valence quark-diquark pair and sea quark-antiquark pairs, distributed around the center of nucleon according to two-dimensional Gauss distribution. The number of sea pairs is distributed according to Poisson law. An elementary interaction is realized in the model of color dipoles $[15,16]$. Then it is assumed, that if there is a collision between two dipoles, two quark-gluon strings are stretched between the ends of the dipoles, and the process of string fragmentation gives observable particles.

It is assumed that each string produces the observed hadrons uniformly distributed in rapidity between the string rapidity ends $y_{\min }$ and $y_{\max }[9,10]$, with the mean number of charged particles per rapidity $\mu_{0}$, and the emission is happing independently in each rapidity interval, with Poisson distribution. Each parton can interact with another one only once, forming a pair of quark-gluon strings, hence, producing particles. 
The transverse position of a string is assigned to the arithmetic mean of the transverse coordinates of the partons at the ends of the string. Due to the finite transverse size of the strings they overlap, that in the framework of string fusion model [11-12] gives a source with higher tension. According to string fusion approach, the mean multiplicity of charged particles and mean $p_{t}$ originated from the cell where $k$ strings are overlapping are the following:

$$
\langle\mu\rangle_{k}=\mu_{0} \sqrt{k},\left\langle p_{t}\right\rangle_{k}=p_{0} \sqrt[4]{k}
$$

Here $\mu_{0}$ and $p_{0}$ are mean charged multiplicity from one single string per rapidity unit and mean transverse momentum from one single string. For the overlapping of the strings of a finite rapidity length, the rapidity space is divided into regions where the number of overlapped strings is an integer, and they are processed separately. All parameters of the model are constrained from the data on inelastic cross section and multiplicity in $\mathrm{pp}, \mathrm{p}-\mathrm{Pb}$ and $\mathrm{Pb}-\mathrm{Pb}$ collisions [17].

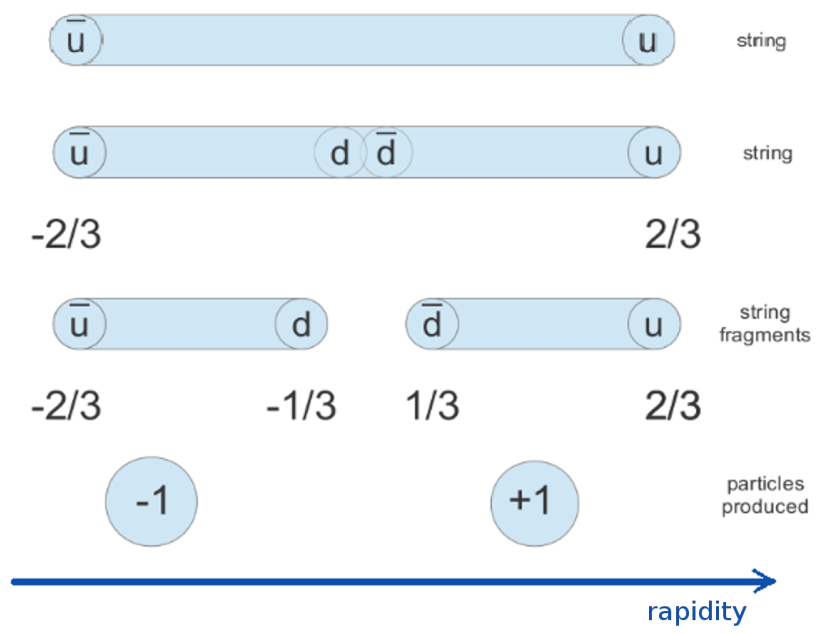

Figure 1: Rapidity string decay and the charge distribution.

The charge differentiation of the particles in this model is shown in figure 1. If one neglects the production of double charge at string fragmentation, each emitted particle can change the charge going along rapidity only by 0 or 1 (emitting of neutral or charged pion). After throwing away the neutral particles, we see that the charge of particles emitted from one string (or a cluster of fused strings) must alternate between 1 to -1 along the rapidity axis. This procedure is implemented for each string or cluster of fused string.

\section{Observables}

For the matter of study the charge fluctuations, we used dynamical fluctuation measure, defined [18] as

$$
v_{\mathrm{dyn}}=\left\langle\left(\frac{N_{+}}{\left\langle N_{+}\right\rangle}-\frac{N_{-}}{\left\langle N_{-}\right\rangle}\right)^{2}\right\rangle-\left(\frac{1}{\left\langle N_{+}\right\rangle}+\frac{1}{\left\langle N_{-}\right\rangle}\right) .
$$


The second term in (2) represents so-called static fluctuation, which appears is no dynamical effects, and this value is subtracted.

More robust value is dynamical fluctuation multiplied by the total number of charged particles: $\quad N_{\mathrm{ch}} v_{\text {dyn }}=\left\langle N_{+}+N_{-}\right\rangle v_{\text {dyn }}$. This value appears as strongly intensive, it depends neither on mean media volume, nor the variance of the volume [19-22].

We also define the correlation coefficient of long range (forward-backward) correlations [23-24] between observables in two separated rapidity windows as follows:

$$
b=\frac{\langle B F\rangle-\langle B\rangle\langle F\rangle}{\left\langle F^{2}\right\rangle-\langle F\rangle^{2}},
$$

where each of $(B, F)$ are the difference between the number of positively and negatively charged particles in each rapidity intervals.

\section{Results}

We performed the calculation of net charge fluctuations and correlations in the Monte Carlo model for $\mathrm{Pb}-\mathrm{Pb}$ collisions at $\sqrt{s_{N N}}=2.76 \mathrm{TeV}$.

\subsection{Net charge fluctuation}

Figure 2. shows the dynamical charge fluctuation, calculated in the model with and without string fusion. The results demonstrates that the model describes qualitatively and quantitatively the behavior of $v_{\text {dyn }}$ with centrality. The inclusion of the string fusion improves the agreement with the experimental data [18].
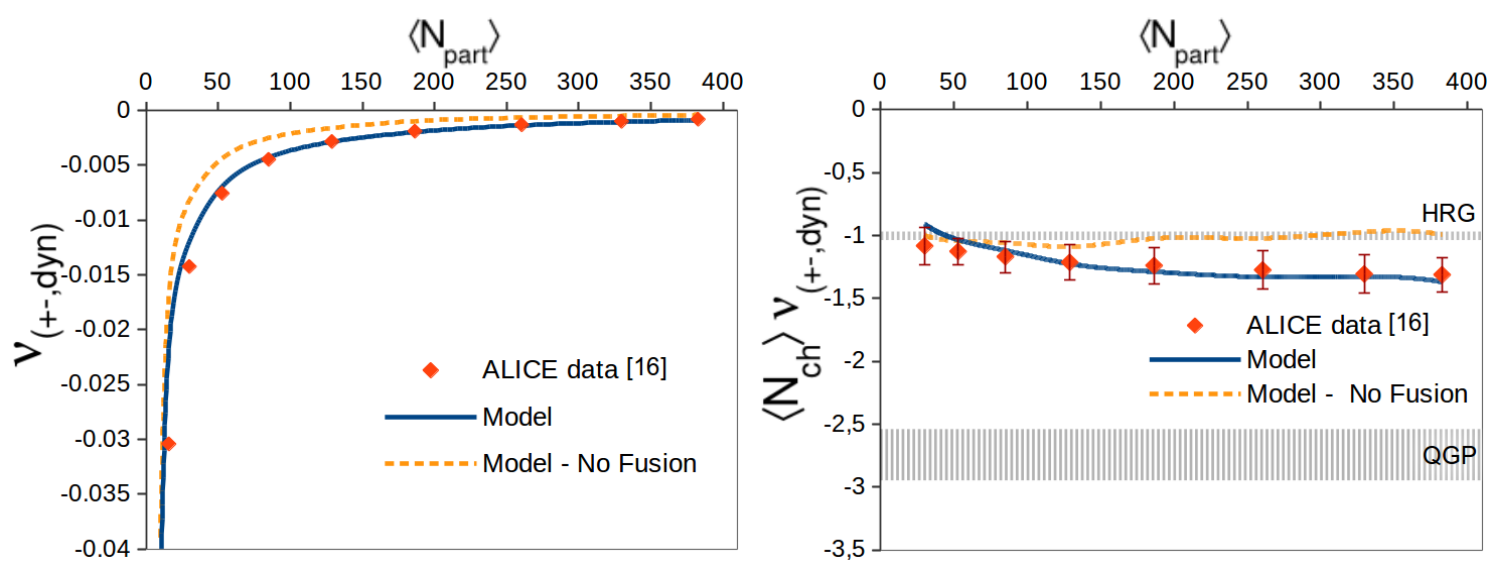

Figure 2: Dynamical charge fluctuation $v_{\mathrm{dyn}}$ as a function of centrality (number of participant nucleons) for $\mathrm{Pb}-\mathrm{Pb}$ collisions at $2.76 \mathrm{TeV}$, calculated in the Monte Carlo model with string fusion and compared with experimental data [18]. The solid line corresponds to the model with string fusion, dashed - without fusion. The right panel shows $v_{\text {dyn }}$ multiplied by multiplicity. Areas that correspond to hadron resonance gas (HRG) and quark-gluon plasma (QGP) are hatched. 
The results of the robust value, shown in the right panel of figure 2 demonstrates the smooth transition from the value, corresponding to hadron resonance gas [25], towards the range of quark-gluon plasma (however it doesn't reach the QGP level, as well as the experimental data). The results without string fusion stay constant at the level of HRG.

\subsection{Net charge forward-backward correlation}

In order to provide a more delicate observable, that can be sensitive to the details of string collectivity, we calculated the introduced before [26] net charge correlation coefficient. The results are shown in figure 3. The calculation shows that at the LHC energy the correlation coefficient is always negative, its absolute value decreases with the increase of the rapidity gap, and it reaches the value close to zero at the separation of about 1 rapidity unit. The results shows weak dependence on the centrality or on the width of the rapidity bin $(\delta \eta)$.

One can argue that at higher rapidity gap, or at the lower energies (SPS or NICA) the transition between the negative and positive correlation coefficient can be observed. At non-zero net-baryon (and net-charge) density the positive forward-backward correlations can occur due to large event-by-event variance of this net-charge density. These predictions can be checked at the current and future experimental detectors (STAR at RHIC, NA61/SHINE at SPS, MPD at NICA).
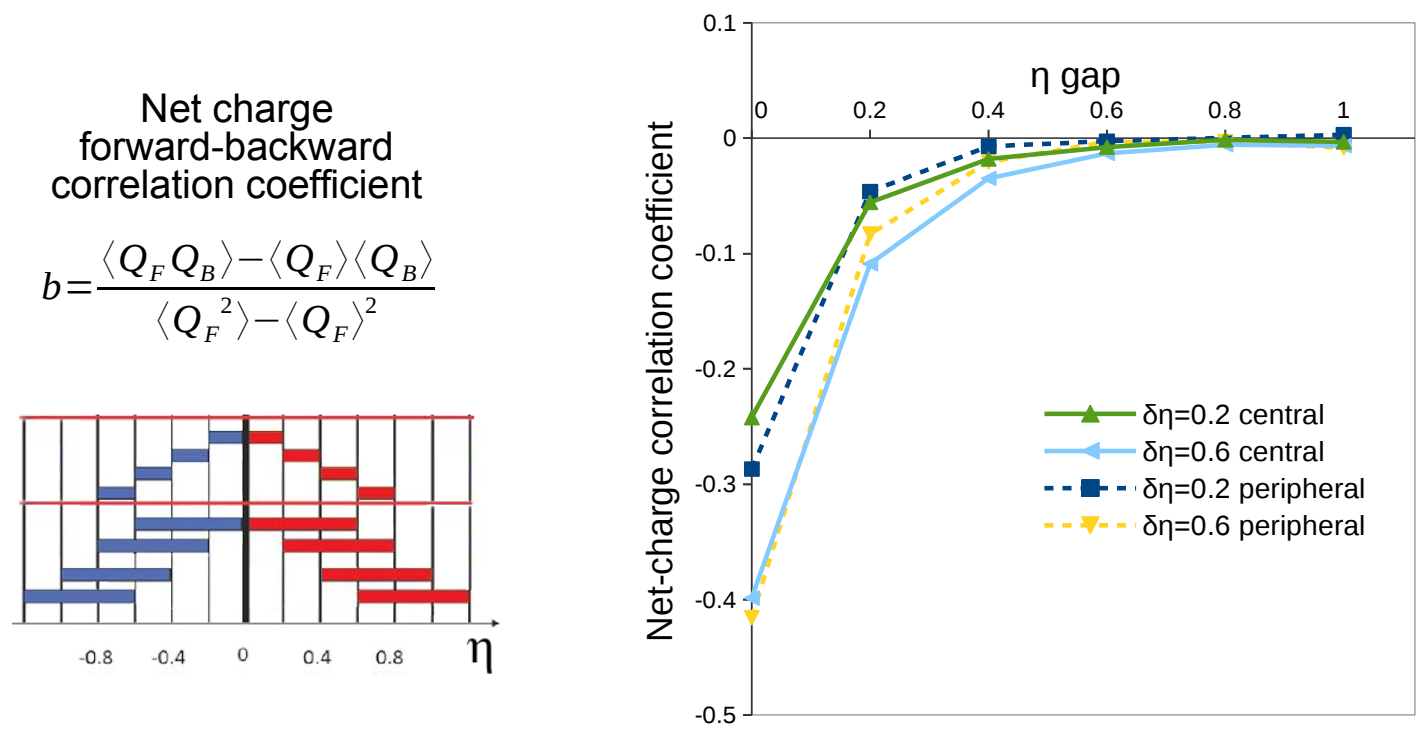

Figure 3: The dependence of the correlation coefficient of the net charge on the gap between the windows (the formula and configuration of the windows are shown on the left panel). Calculations are made for two centrality ranges and different widths of a rapidity window for $\mathrm{Pb}-\mathrm{Pb}$ collisions at $2.76 \mathrm{TeV}$. 


\section{Conclusions and outlook}

The string decay model, which includes finite rapidity width of a string, string fusion, and local charge conservation, has been developed. The Monte Carlo model with string fusion reasonably describes the centrality dependence of the net-net charge fluctuation (without any parameter tuning). The version of the model without string fusion cannot agree with the experimental data. The predictions for net-charge forward-backward correlation coefficient have been obtained.

Further studies should also include taking into account experimental cuts on the transverse momentum of the produced particles, implementation of the resonance production and decay, application at different energies (SPS, RHIC and NICA) and study of mixed type correlations involving multiplicity, pt, charge, strangeness, baryon number etc. Some of these variables, especially in pp collisions, can be calculated in the extended multi-pomeron model [27-29] and other models with string collectivity.

\section{Acknowledgements}

The research was supported by the grant of the Russian Foundation for Basic Research (project 18-32-01055 mol_a).

\section{References}

[1] S. Jeon and V. Koch, Phys. Rev. Lett. 85 (2000) 2076.

[2] M. Asakawa, U. Heinz, and B. Müller, Phys. Rev. Lett. 85 (2000) 2072.

[3] S. Jeon and V. Koch, Phys. Rev. Lett. 85, 2076 (2000).

[4] C. Pruneau, S. Gavin and S. Voloshin, Phys. Rev. C 66, 044904 (2002).

[5] M. Gazdzicki, M. I. Gorenstein, M. Mackowiak-Pawlowska, Phys. Rev. C 88, 024907 (2013)

[6] M. Asakawa, U. Heinz and B. Müller, Phys. Rev. Lett. 85, 2072 (2000).

[7] E.V. Shuryak, M.A. Stephanov, Phys. Rev. C 63, 064903 (2001).

[8] A. Titov, V. Vechernin, PoS (Baldin ISHEPP XXI) 047, 2012.

[9] V. N. Kovalenko. Phys. Atom. Nucl. 76, 1189 (2013).

[10] V. Kovalenko, V. Vechernin, PoS (Baldin ISHEPP XXI) 072.

[11] M.A. Braun, C. Pajares, and V.V. Vechernin, Phys. Let. B 493, 54 (2000).

[12] M. Braun, R. Kolevatov, C. Pajares, and V. Vechernin, Eur. Phys. J. C 32, 535 (2004)

[13] V. Kovalenko, V. Vechernin, EPJ Web of Conferences 66, 04015 (2014).

[14] I. Altsybeev, KnE Energy and Physics ICPPA2017, 304-312, 2018, arXiv:1711.04844 [nucl-ex].

[15] C. Flensburg, G. Gustafson, L. Lonnblad, Eur. Phys. J. C 60, 233 (2009), arXiv: 0807.0325 [hep-ph]

[16] G. Gustafson, Acta Phys. Polon. B 40, 1981 (2009), arXiv: 0905.2492 [hep-ph] 
[17] V. Kovalenko, PoS (QFTHEP2013), 052 (2013)

[18] B. Abelev et al. (ALICE Collaboration), Phys. Rev. Lett. 110, 152301 (2013), arXiv: 1207.6068 [nucl-ex]

[19] M.I. Gorenstein, M. Gazdzicki, Phys. Rev. C 84, 014904 (2011), arXiv: 1101.4865 [nucl-th]

[20] M. Gazdzicki, M. Gorenstein, M. Mackowiak-Pawlowska, Phys. Rev. C 88, 024907 (2013), arXiv: 1303.0871 [nucl-th]

[21] E.V. Andronov, Theor. Math. Phys. 185, 1383 (2015) [Teor. Mat. Fiz. 185, no. 1, 28 (2015)]

[22] E. Andronov, V. Vechernin, arXiv: 1808.09770 [hep-ph] (2018)

[23] Vechernin, V.V. Nucl.Phys. A939 (2015) 21-45 arXiv:1210.7588 [hep-ph]

[24] V. Vechernin, EPJ Web of Conferences, 164, 07021 (2017).

[25] Sangyong Jeon and Volker Koch, "Event by event fluctuations", In Quark Gluon Plasma 3, Ed. R.C. Hwa andX.N. Wang, (2004) 430, arXiv: hep-ph/0304012.

[26] D. Neverov, PoS (Baldin ISHEPP XXII) 051 (2015).

[27] N. Armesto, D. A. Derkach, G. A. Feofilov, Phys. Atom. Nucl. 71, 2087 (2008).

[28] E. Bodnia, D. Derkach, G. Feofilov, V. Kovalenko, A. Puchkov, PoS (QFTHEP 2013) 060 (2013), arXiv:1310.1627 [hep-ph].

[29] E. O. Bodnia, V. N. Kovalenko, A. M. Puchkov, G. A. Feofilov, AIP Conf. Proc. 1606, 273-282 (2014), arXiv:1401.7534 [hep-ph]. 